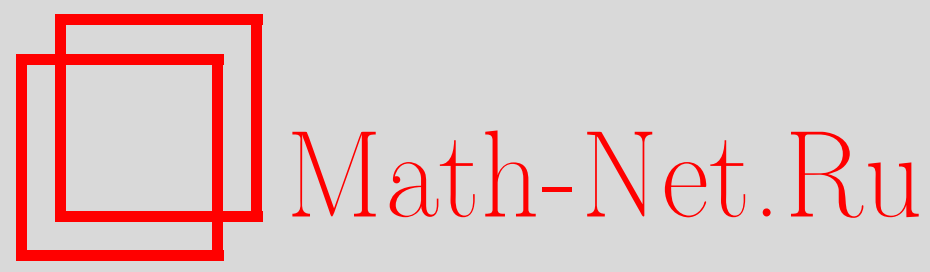

М. И. Нейман-заде, А. А. Шкаликов, Операторы Шрёдингера с сингулярными потенциалами из пространств мультипликаторов, Матем. заметки, 1999, том 66, выпуск 5, 723733

DOI: https://doi.org/10.4213/mzm1216

Использование Общероссийского математического портала Math-Net.Ru подразумевает, что вы прочитали и согласны с пользовательским соглашением http://www.mathnet.ru/rus/agreement

Параметры загрузки:

IP : 52.87 .193 .239

26 апреля 2023 г., 18:36:39

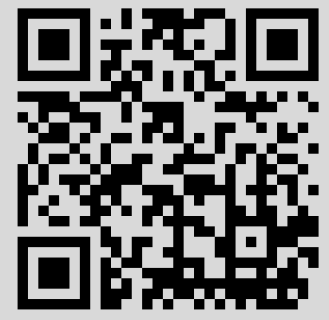




\title{
ОПЕРАТОРЫ ШРЁДИНГЕРА С СИНГУЛЯРНЫМИ ПОТЕНЦИАЛАМИ ИЗ ПРОСТРАНСТВ МУЛЬТИПЛИКАТОРОВ
}

\author{
М. И. Нейман-заде, А. А. Шкаликов
}

В работе изучаются операторы Шрёдингера $T+Q$, где $T=-\Delta-$ оператор Лапласа, а $Q$ - оператор умножения на обобщенную функцию. Рассматриваются также обобщения для случая полигармонического оператора $T=(-\Delta)^{n}$.

Библиограбоя: 20 названий.

Введение. В работе изучаются операторы Шрёдингера $T+Q$, где $T=-\Delta$ - оператор Лапласа, а $Q$ - оператор умножения на обобщенную функцию. Рассматриваются также обобщения для случая полигармонического оператора $T=(-\Delta)^{m}$. Операторы такого типа появились в физических работах $30-$ х годов в связи с задачей рассеяния нейтральных частиц на ядре, когда взаимодействие является сильным на малых расстояниях и пренебрежимо малым на средних и больших (см. [1]). Модельньм потенциалом такого взаимодействия является $\delta$-функция Дирака.

Математическое исследование оператора $-\Delta+\mu \delta(x)$ было предпринято Березиным и Фаддеевым [2], Минлосом и Фаддеевым [3], Березиным [4]. В работах [2], [3] оператор $-\Delta+\mu \delta(x)$ понимался как расширение оператора $T_{0}=-\Delta$ с областью $\mathcal{D}\left(T_{0}\right)=$ $C_{0}^{\infty}\left(\mathbb{R}^{3} \backslash\{0\}\right)$. Эта тема вызвала большое число работ. В основном изучались сингулярные потенциалы, сосредоточенные на многообразиях и дискретных наборах точек. Укажем здесь работы [5]-[9] и книги [10], [11], где можно найти более полную библиографию. Недавно начал развиваться метод [4], основанный на резольвентньх тождествах.

Знакомясь с этой темой, мы не нашли работ, где исследовались сингулярныепотенциалы с носителями на множествах ненулевой меры, кроме статей [12], [13], где рассматривался потенциал $1 / x$ в $L_{2}\left[\mathbb{R}^{1}\right]$. Возникает задача: для каких сингулярных функций $q(x)$ можно корректно определить оператор $-\Delta+q(x) ?$

Естественньй подход к решению этой задачи - воспользоваться методом квадратичных форм. Необходимое условие, чтобы этот метод работал, состоит в следующем: функция $q$ должна быть мультипликатором из пространства $H^{1}\left(\mathbb{R}^{n}\right)$ в $H^{-1}\left(\mathbb{R}^{n}\right)$, т.е. умножение на нее есть ограниченньй оператор из $H^{1}$ в $H^{-1}$. Однако, исследований на тему, когда функция является мультипликатором в этих пространствах, мы не обнаружили, за исключением одного результата в книге [14], который будет использоваться в работе (см. теорему 6). Конечно, задача описания пространства мультипликаторов

Работа выполнена при поддержке Российского фонда фундаментальных исследований, гранты № 98-01-01000 и № 96-15-96091. 
$M\left[H^{m} \rightarrow H^{-m}\right]$ интересна не только с точки зрения теории операторов Шрёдингера, но представляет и самостоятельньй интерес.

Имеется еще одна важная задача: если оператор $-\Delta+q$ с сингулярным потенциалом $q$ уже определен, то можно ли его в некотором смысле приблизить операторами с гладкими потенциалами, по крайней мере так, чтобы спектры приближающих операторов были близки к спектру исходного?

Целью заметки является решение перечисленных здесь задач. В первом разделе мы даем определение суммы операторов в общих терминах, основанное на методе квадратичных форм. Ядром метода является известная КЛМН-теорема (см. $[15$, гл. Х]). Здесь в нужной нам форме приводятся ее полезные обобщения. Отметим, что подробньй анализ возможности определения суммы операторов дан в недавней работе [16]. Во втором разделе мы приводим абстрактное обобщение следующего результата: если последовательность функций $q_{n}$ сходится к $q$ в пространстве мультипликаторов, то последовательность операторов $-\Delta+q_{n}$ сходится к $-\Delta+q$ в смысле равномерной резольвентной сходимости, и имеет место сходимость спектров. В третьем разделе мы выделяем два важных подпространства в пространстве мультипликаторов $M\left[H^{\theta} \rightarrow H^{-\theta}\right]$ и приводим достаточные условия принадлежности функций этим подпространствам. В четвертом разделе мы формулируем итоговый результат и приводим примеры.

1. Определение суммы симметрических операторов. Пусть $T$ - самосопряженньй полуограниченньй оператор в гильбертовом пространстве $\mathfrak{H}$. Далее удобно считать, что $T \geqslant I$, где $I$ - тождественньй оператор. С оператором $T$ свяжем шкалу гильбертовых пространств $\mathfrak{H}_{\theta}$. При $\theta \geqslant 0$ положим $\mathfrak{H}_{\theta}=\mathcal{D}\left(T^{\theta / 2}\right)$ с нормой $\|x\|_{\theta}=$ $\left\|T^{\theta / 2} x\right\|$, а $\mathfrak{H}_{-\theta}$ определим как пространство, дуальное к $\mathfrak{H}_{\theta}$ по отношению к $\mathfrak{H}=\mathfrak{H}_{0}$. В частности, $\mathfrak{H}_{2}=\mathcal{D}(T)$. Через $(\cdot, \cdot)$ обозначаем скалярное произведение в $\mathfrak{H} ;$ это же обозначение сохраняем для дуального скалярного произведения элементов $\mathfrak{H}_{-1}$ и $\mathfrak{H}_{1}$, полагая

$$
(x, y)=\left(T^{-1 / 2} x, T^{1 / 2} y\right), \quad x \in \mathfrak{H}_{-1}, \quad y \in \mathfrak{H}_{1} .
$$

Оператор $Q: \mathfrak{H}_{1} \rightarrow \mathfrak{H}_{-1}$ (рассматриваемьй как оператор из $\mathfrak{H}_{1}$ в $\mathfrak{H}_{-1}$ ) назовем симметрическим, если порожденная им в смысле дуального скалярного произведения квадратичная форма $(Q v, v)$ является вешественной. По определению сопряженные пространства $\mathfrak{H}_{1}^{*}$ и $\mathfrak{H}_{-1}^{*}$ совпадают с $\mathfrak{H}_{-1}$ и $\mathfrak{H}_{1}$. Поэтому сопряженный оператор $Q^{*}$ действует также из $\mathfrak{H}_{1}$ в $\mathfrak{H}_{-1}$ (см. определение в $[17$, гл. III, $\left.\S 5.5]\right)$, и в случае симметрического оператора $Q$ имеем $Q \subset Q^{*}$. Если $Q=Q^{*}$, то $Q$ назьвают самосопряжсенным. Следующий результат эквивалентен известной КЛМН-теореме (см. [17, гл. II, § 1.6], [15, гл. $\mathrm{X}]):$ Если симметрический оператор $Q: \mathfrak{H}_{1} \rightarrow \mathfrak{H}_{-1}$ определен на всем $\mathfrak{H}_{1} u$ подчинен оператору $T$ в смысле форм с $T$-гранью $\alpha<1$, m.е.

$$
|(Q v, v)|<\alpha\|v\|_{1}^{2}+\beta\|v\|^{2}, \quad v \in \mathfrak{H}_{1}, \quad \beta=\mathrm{const},
$$

то квадратичная форма $((T+Q) v, v)$, определенная на $\mathfrak{H}_{1}$, замкнута и полуограничена. Первую теорему о представлении (см. [17, гл. VI, § 2.1]) в этой ситуации можно переформулировать в следующем виде. Onepamop $L=T+Q: \mathfrak{H}_{1} \rightarrow \mathfrak{H}_{-1}$ самосопряжен, а его сужение на область

$$
\mathcal{D}(L)=\left\{v \in \mathfrak{H}_{1} \mid T v+Q v \in \mathfrak{H}\right\}
$$

является самосопряженным полуограниченным оператором в $\mathfrak{H}$.

Полезно иметь в виду следующее обобщение сформулированного результата. 
Tеорема 1. Пусть оператор $Q: \mathfrak{H}_{1} \rightarrow \mathfrak{H}_{-1}$ определен на всем $\mathfrak{H}_{1}$ и симметричен. Если найдутся числа $\alpha<1, \beta>0$ такие, что

$$
(Q v, v) \geqslant-\alpha\|v\|_{1}^{2}-\beta\|v\|^{2}, \quad v \in \mathfrak{H}_{1},
$$

то оператор $T+Q: \mathfrak{H}_{1} \rightarrow \mathfrak{H}_{-1}$ является самосопряженным, а его сужение $T \widetilde{+} Q$ на область

$$
\mathcal{D}(T \widetilde{+} Q)=\left\{v \in \mathfrak{H}_{1} \mid T v+Q v \in \mathfrak{H}\right\}
$$

является полуограниченным самосопряженным оператором в $\mathfrak{H}$. Другими словами: определенная на $\mathfrak{H}_{1}$ квадратичная форма $((T+Q) v, v)$ замкнута, а ассоииированный $c$ этой формой оператор $T \widetilde{+} Q$ полуограничен и самосопряжен $в$ $\mathfrak{H}$.

ДокАЗАТЕльство. Так как симметрический оператор $Q$ определен на всем $\mathfrak{H}_{1}$, то он совпадает со своим сопряженным $Q^{*}$, а потому замкнут. Согласно теореме о замкнутом графике $[17$, гл. III, $\S 5.4]$ оператор $Q: \mathfrak{H}_{1} \rightarrow \mathfrak{H}_{-1}$ ограничен. Поэтому найдется постоянная $\gamma>0$ такая, что $|(Q v, v)| \leqslant \gamma\|v\|_{1}^{2}$. В силу оценки $(1)$ имеем

$$
(1-\alpha)\|v\|_{1}^{2} \leqslant((T+Q+\beta) v, v) \leqslant(\gamma+1)\|v\|_{1}^{2}+\beta\|v\|_{1}^{2}, \quad v \in \mathfrak{H}_{1} .
$$

Теперь доказательство завершается стандартньм путем, так же как в КЛМН-теореме. Можно воспользоваться также методом продолжения по параметру для семейства операторов $T+\varkappa Q, 0 \leqslant \varkappa \leqslant 1$.

ТЕОрема 2. Пусть линеал $D_{0}$ является плотным подмножеством в $\mathfrak{H}_{1}$, оператор $S_{0}: \mathfrak{H}_{1} \rightarrow \mathfrak{H}_{-1}$ удовлетворяет условию

$$
\left(S_{0} v, v\right) \geqslant(T v, v)-\beta(v, v) \quad \forall v \in D_{0}, \quad \beta=\text { const }
$$

a оператор $Q: \mathfrak{H}_{1} \rightarrow \mathfrak{H}_{-1}$ определен на всем $\mathfrak{H}_{1}$ и удовлетворяет оценке (1). Пусть $S$ - расширение по Фридрихсу полуограниченного оператора $S_{0}$, a $\mathfrak{H}_{S}$ область определения замыкания квадратичной формы $\left(S_{0} v, v\right)$. Тогда $\mathfrak{H}_{S} \subset \mathfrak{H}_{1}$, оператор $S+Q: \mathfrak{H}_{S} \rightarrow \mathfrak{H}_{S}^{*}$ самосопряжен, а его сужение $S \widetilde{+} Q$ на область

$$
\mathcal{D}(S \widetilde{+} Q)=\left\{v \in \mathfrak{H}_{S} \mid S v+Q v \in \mathfrak{H}\right\}
$$

является самосопряженным полуограниченным оператором в $\mathfrak{H}$.

ДокАЗАТЕЛЬСТво. Из условий теоремы имеем оценку

$$
\left(\left(S_{0}+Q+2 \beta\right) v, v\right) \geqslant(1-\alpha)\|v\|_{1}^{2}, \quad v \in D_{0} .
$$

Следовательно, квадратичная форма, порожденная оператором $S_{0}+Q$, полуограничена и допускает замыкание на некоторую область $\mathfrak{H}_{S}$. Из оценки (3) и плотности линеала $D_{0}$ в $\mathfrak{H}_{1}$ заключаем $\mathfrak{H}_{S} \subset \mathfrak{H}_{1}$. Остальные утверждения вытекают из определений.

ЗАмЕчаниЕ 1. Сформулированные теоремы отличаются лиш по форме. С одной стороны, теорема 1 есть частный случай теоремы 2 при $S_{0}=T$. С другой стороны, теорему 2 легко вывести из теоремы 1 , взяв вместо $T$ оператор $S+\beta$. При этом пространства $\mathfrak{H}_{1}$ и $\mathfrak{H}_{-1}$ заменяются на $\mathfrak{H}_{S}$ и $\mathfrak{H}_{S}^{*}$. 
ЗАмЕчАниЕ 2. Теорему 2 моожно переформулировать по-другому, если мы не хотим ограничиваться расширением по Фридрихсу. В формулировке можно предположить с самого начала, что $S$ - самосопряженный оператор такой, что область определения $\mathfrak{H}_{S}$ ассоциированной с ним квадратичной формы вложена в $\mathfrak{H}_{1}$ и вьполнена оценка (3) для $v \in \mathfrak{H}_{S}$. В этом случае утверждение теоремы сохраняет силу.

2. Приближения операторных сумм. Резольвентная сходимость и сходимость спектров. В рассматриваемых ниже примерах в качестве оператора $Q$ выступают операторы умножения на обобщенную функцию $q$. Если сумма операторов уже определена, то правомерно ли (например, в компютерных вычислениях) заменять сингулярную функцию $q_{n}$, близкую к $q$ в смысле сходимости в некотором пространстве обобщенных функций? С этой точки зрения представляют интерес излагаемые ниже результаты.

Сначала напомним некоторые определения. Пусть $\left\{A_{n}\right\}$ - последовательность замкнутых операторов в банаховом пространстве $\mathcal{X}$. Будем говорить, что операторы $A_{n}$ сходятся при $n \rightarrow \infty$ к оператору $A$ в смысле равномерной (сильной) резольвентной сходимости и писать $A_{n} \stackrel{R}{\Longrightarrow} A\left(A_{n} \stackrel{R}{\longrightarrow} A\right)$, если найдется число $\zeta \in \mathbb{C}$ такое, что $\zeta \in \rho(A) \cap \rho\left(A_{n}\right)$ для всех достаточно больших $n$ и $\left(A_{n}-\zeta\right)^{-1}$ равномерно (сильно) сходятся к $(A-\zeta)^{-1}$. Известно [17, гл. VIII, теорема 2.25$]$, что равномерная резольвентная сходимость влечет сходимость спектров $\sigma\left(A_{n}\right)$ к $\sigma(A)$ сверху. В случае гильбертова пространства и самосопряженных операторов $A_{n}$ не только равномерная, но и сильная резольвентная сходимость влечет сходимость спектров снизу [17, гл. VIII, теорема 1.14]. Поэтому в случае самосопряженных операторов $A_{n}$ имеем следующий результат: $e c л u$ $A_{n} \stackrel{R}{\Longrightarrow} A, \operatorname{mo} \sigma\left(A_{n}\right) \rightarrow \sigma(A)$.

ТЕОрема 3. Пусть операторы $S_{0}$ и $Q$ удовлетворяют условиям теоремы 2, a $S$ - расширение по Фридрихсу оператора $S_{0}$. Предположим, что операторы $Q_{n}: \mathfrak{H}_{1} \rightarrow \mathfrak{H}_{-1}$ определены на всем $\mathfrak{H}_{1}$, для них выполнена оченка (1) и для всех $v \in \mathfrak{H}_{1}$

$$
\left\|\left(Q-Q_{n}\right) v\right\|_{-1} \rightarrow 0 \quad n p u \quad n \rightarrow \infty .
$$

Тогда $S \widetilde{+} Q_{n} \stackrel{R}{\longrightarrow} S \widetilde{+} Q$. Если оператор $T^{-1}$ компактен в $\mathfrak{H}$ или соотношение (4) выполняется равномерно для $v$, удовлетворяющих условию $\|v\|_{1}=1$, то $S \widetilde{+}$ $Q_{n} \stackrel{R}{\Longrightarrow} S \widetilde{+} Q$. В этом случае имеет место сходимость спектров

$$
\sigma\left(S \widetilde{+} Q_{n}\right) \rightarrow \sigma(S \widetilde{+} Q)
$$

ДокАЗАТЕЛЬСтво. Согласно замечанию 1 достаточно доказать теорему для случая $S=T$. Пусть $T^{1 / 2}-$ квадратньй корень из $T$. Очевидно, $T^{1 / 2}: \mathfrak{H}_{\theta} \rightarrow \mathfrak{H}_{\theta-1}$ есть изоморфизм. Поэтому операторы

$$
K_{n}:=T^{-1 / 2} Q_{n} T^{-1 / 2}: \mathfrak{H} \rightarrow \mathfrak{H}
$$

ограничены, причем из условий теоремы и оценки (2) получаем

$$
-\alpha\|x\|^{2}-\beta\left\|T^{-1} x\right\|^{2} \leqslant\left(K_{n} x, x\right) \leqslant \gamma\|x\|^{2}+\beta\left\|T^{-1} x\right\|^{2},
$$

где $\alpha<1$. Следовательно, операторы

$$
Z_{n}:=I-K_{n}-\rho T^{-1}
$$


обратимы при $\rho<-\beta$, причем они сами и обратные к ним равномерно ограничены. Имеем

$$
\left(T+Q_{n}-\rho\right)^{-1}=T^{-1 / 2} Z_{n}^{-1} T^{-1 / 2},
$$

где левая часть понимается как оператор из $\mathfrak{H}_{-1}$ в $\mathfrak{H}_{1}$. Если в обозначениях (5) и (6) опустить индекс $n$, то последнее равенство остается справедливым для $Q$ вместо $Q_{n}$. Тогда имеем

$$
\begin{aligned}
\left(T+Q_{n}-\rho\right)^{-1}-(T+Q-\rho)^{-1} & =\left(T+Q_{n}-\rho\right)^{-1}\left(Q-Q_{n}\right)(T+Q-\rho)^{-1} \\
& =T^{-1 / 2} Z_{n}^{-1}\left[T^{-1 / 2}\left(Q-Q_{n}\right) T^{-1 / 2}\right] Z^{-1} T^{-1 / 2}
\end{aligned}
$$

Здесь все множители - ограниченные операторы в $\mathfrak{H}$, причем $Z_{n}^{-1}$ равномерно ограничены. Условие (4) влечет $T^{-1 / 2}\left(Q-Q_{n}\right) T^{-1 / 2} \rightarrow 0$ в пространстве $\mathfrak{H}$. Заметим, что $\left(T \widetilde{+} Q_{n}-\rho\right)^{-1}$ есть сужение оператора $\left(T+Q_{n}-\rho\right)^{-1}$; то же верно, если $Q_{n}$ заменить на $Q$. Поэтому $T \widetilde{+} Q_{n} \stackrel{R}{\longrightarrow} T \widetilde{+} Q$. Если оператор $T^{-1}$ компактен, то тем же свойством обладает оператор $T^{-1 / 2}$. Умножение как справа, так и слева на компактньй оператор переводит сильную сходимость в равномерную. Сходимость также равномерна, если условие (4) вьполняется равномерно на единичной сфере в $\mathfrak{H}_{1}$. Но равномерная резольвентная сходимость, как уже отмечалось, влечет сходимость спектров.

Tеорема 4. Пусть операторы $S_{0}$ u $S$ такие же, как в теореме 2, а оператор $Q: \mathfrak{H}_{1} \rightarrow \mathfrak{H}_{-1}$ симметричен и компактен. Тогда справедливо утвержсдение теоремы 2, более того, непрерывные спектры операторов $S \widetilde{+} Q$ и $S$ совпадают.

ДокАЗАтЕльство. Условие компактности оператора $Q: \mathfrak{H}_{1} \rightarrow \mathfrak{H}_{-1}$ влечет условие его подчиненности оператору $T$ в смысле форм с произвольно малой $T$-гранью $\alpha>0$. Поэтому утверждение теоремы 2 справедливо. Далее, спектр оператора $S+Q: \mathfrak{H}_{S} \rightarrow$ $\mathfrak{H}_{S}^{*}$ совпадает со спектром его сужения $S \widetilde{+} Q: \mathcal{D}(S \widetilde{+} Q) \rightarrow \mathfrak{H}$. То же верно и для непрерьвных компонент спектра. Согласно определению точка $\rho$ не принадлежит непрерывному спектру оператора $S+Q$, если и только если оператор $S+Q-\rho: \mathfrak{H}_{S} \rightarrow \mathfrak{H}_{S}^{*}$ является фредгольмовым. Но $\mathfrak{H}_{S}$ непрерывно вложено в $\mathfrak{H}_{1}$. Поэтому если $Q: \mathfrak{H}_{1} \rightarrow \mathfrak{H}_{-1}$ компактен, то $Q: \mathfrak{H}_{S} \rightarrow \mathfrak{H}_{S}^{*}$ также компактен. Согласно второй теореме устойчивости [17, гл. IV] компактные слагаемые не влияют на свойство оператора быть фредгольмовып. Поэтому $\sigma_{c}(S+Q)=\sigma_{c}(S \widetilde{+} Q)=\sigma_{c}(S)$. Теорема доказана.

3. Мультипликаторы в пространствах Соболева. Пусть $H_{p}^{\theta}\left(\mathbb{R}^{n}\right), \theta \in \mathbb{R}, p \geqslant$ $1,-$ пространства Соболева, норма в которых определена равенством

$$
\|u\|_{H_{p}^{\theta}}=\left\|F^{-1}\left(1+|x|^{2}\right)^{\theta / 2} F u\right\|_{L_{P}\left(\mathbb{R}^{n}\right)}
$$

где $F$ - преобразование Фурье. Далее полагаем $\mathfrak{H}_{\theta}=H_{2}^{\theta}\left(\mathbb{R}^{n}\right)$. Через $D$ обозначаем пространство основных функций $D:=C_{0}^{\infty}\left(\mathbb{R}^{n}\right)$.

Мы будем изучать мультипликаторы из пространства $\mathfrak{H}_{\theta}$ в $\mathfrak{H}_{-\theta}$. Формальное определение следующее: $\varphi \in D^{\prime}$ мультипликатор, если для всех $g \in \mathfrak{H}_{\theta}$ произведение $\varphi g \in$ $\mathfrak{H}_{-\theta}$. Однако произведение на обобщенную функцию $\varphi$ определено лишь для $g \in C^{\infty}$, поэтому это определение подлежит коррекции. Функцию $\varphi \in D^{\prime}$ назовем мультипликатором из $\mathfrak{H}_{\theta}$ в $\mathfrak{H}_{-\theta}$, если существует постоянная $C$ такая, что

$$
|(\varphi g, f)|=|(\varphi, \bar{g} f)| \leqslant C\|g\|_{\theta}\|f\|_{\theta} \quad \forall f, g \in D .
$$


Если это условие вьполнено, то билинейная форма $(\varphi g, f)$ продолжается по непрерьвности для всех $f, g \in \mathfrak{H}_{\theta}$. Если элемент $g$ фиксирован, то $\varphi_{g}(f):=(\varphi g, f)$ является антилинейным непрерывным функционалом в $\mathfrak{H}_{\theta}$, т.е. принадлежит $\mathfrak{H}_{-} \theta$. Произведение $\varphi g$ теперь можно положить равным $\varphi_{g}$.

Мультипликатор $\varphi$ определяет ограниченньй линейный оператор $Q_{\varphi}: \mathfrak{H}_{\theta} \rightarrow \mathfrak{H}_{-\theta}$, определяемый равенством $Q_{\varphi} g=\varphi_{g}$. Если $\varphi$ - вещественная функция, то $Q_{\varphi}$ симметричен и определен на всем $\mathfrak{H}_{\theta}$, а потому самосопряжен.

Билинейная форма в гильбертовом пространстве определяется квадратичной формой, поэтому определение (7) можно заменить следующим

$$
|(\varphi f, f)| \leqslant \alpha\|f\|_{\theta}^{2}+\beta\|f\|^{2} \leqslant \alpha^{\prime}\|f\|_{\theta}^{2} .
$$

Нижнюю грань чисел $\alpha^{\prime}$, при которых справедливы эти неравенства, назьвают нормой $\varphi$, а нижнюю грань чисел $\alpha$ (с произвольной константой $\beta$ ) назьвают существенной нормой. Пространство мультипликаторов $M\left[\mathfrak{H}_{\theta} \rightarrow \mathfrak{H}_{-\theta}\right]$, наделенное обычной нормой, является банаховьм пространством. Далее обозначаем его через $M[\theta]$. Через $\|\varphi\|_{M[\theta]}$ и ess $\|\varphi\|_{M[\theta]}$ обозначаем норму и существенную норму элементов $\varphi \in M[\theta]$. Полезно ввести еще понятие существенной нижней грани мультипликатора $\varphi$. Она совпадает с верхней гранью чисел $\gamma$ таких, что

$$
(\varphi f, f) \geqslant \gamma\|f\|_{\theta}^{2}-\beta\|f\|^{2}
$$

при всевозможных $\beta$.

Аналогично определяется пространство мультипликаторов $M\left[\mathfrak{H}_{\theta} \rightarrow \mathfrak{H}\right]$. Оно состоит из функций $\varphi \in L_{2, \text { loc }}$, для которых

$$
|(\varphi f, g)| \leqslant C\|f\|_{\theta}\|g\|_{0} \quad \forall f, g \in D \text {. }
$$

В пространстве $M[\theta]$ полезно выделить подпространства $\stackrel{0}{M}[\theta]$ и $M_{0}[\theta]$. Подпрос0 транство $M[\theta]$ состоит из мультипликаторов $\varphi$, которые можно приблизить с произвольной точностью по норме $M[\theta]$ функциями из основного пространства $D$. Отметим, что умножение на функцию $\varphi \in D$ является не только ограниченным, но и компактным оператором из $\mathfrak{H}_{\theta}$ в $\mathfrak{H}_{-\theta}$. Действительно, если $\varphi \in D$, то носитель $\varphi$ финитен, поэтому утверждение следует из компактности вложения $H_{2}^{\theta}(\Omega) \subset H_{2}^{-\theta}(\Omega)$, где $\Omega$ - произвольная компактная область, содержащая носитель $\varphi$. Если $\left\|Q_{\varphi}\right\|$ - норма оператора $Q_{\varphi}: \mathfrak{H}_{\theta} \rightarrow \mathfrak{H}_{-\theta}$, то из определения следует $\left\|Q_{\varphi}\right\|=\|\varphi\|_{M[\theta]}$. Поэтому если $\varphi \in \stackrel{0}{M[\theta] \text { и }}$ $\varphi_{n} \rightarrow \varphi$ по норме $M[\theta], \varphi_{n} \in D$, то предел $Q_{\varphi}$ компактных операторов $Q_{\varphi_{n}}$ (по норме пространства операторов из $\mathfrak{H}_{\theta}$ в $\mathfrak{H}_{-\theta}$ ) также является компактньп оператором из $\mathfrak{H}_{\theta}$ в $\mathfrak{H}_{-}$. Таким образом, $\stackrel{0}{M}[\theta]$ состоит из компактных мультипликаторов. Подпространство $M_{0}[\theta]$ определим как множество $\varphi \in M[\theta]$, для которых существенная норма равна 0 . Очевидно, $M_{0}[\theta] \supset \stackrel{0}{M}[\theta]$.

Приведем несколько достаточных условий принадлежности функций пространствам 0 $M[\theta]$ и $M_{0}[\theta]$. 
Tеорема 5. Пусть $\theta>n / 2$ (здесь $n-$ число переменных у функиий из пространства $\left.\mathfrak{H}=L_{2}\left(\mathbb{R}^{n}\right)\right)$. Тогда

$$
\mathfrak{H}_{-\theta} \subset \stackrel{0}{M}[\theta]
$$

и справедлива оченка

$$
\|\varphi\|_{M[\theta]} \leqslant C\|\varphi\|_{-\theta}, \quad C=\text { const . }
$$

ДокаЗАТЕЛЬСтво. Известно (см. [14, гл. I, § 1.5] и имеющиеся там ссылки), что при $\theta>n / 2$ пространства $\mathfrak{H}_{\theta}$ образуют алгебру, т.е. условие $f, g \in \mathfrak{H}_{\theta}$ влечет $f g \in \mathfrak{H}_{\theta}$ и

$$
\|f g\|_{\theta} \leqslant C\|f\|_{\theta}\|g\|_{\theta} .
$$

Пусть $\varphi \in \mathfrak{H}_{-\theta}$. Тогда для $f, g \in D$ имеем

$$
|(\varphi f, g)|=|(\varphi, \bar{f} g)| \leqslant\|\varphi\|_{-\theta}\|\bar{f} g\|_{\theta} \leqslant C\|\varphi\|_{-\theta}\|f\|_{\theta}\|g\|_{\theta} .
$$

Следовательно, $\varphi \in M[\theta]$, причем

$$
\|\varphi\|_{M[\theta]} \leqslant C\|\varphi\|_{-\theta} .
$$

По определению пространство $\stackrel{0}{M}[\theta]$ есть замыкание $D$ по норме $M[\theta]$, а $\mathfrak{H}_{-\theta}$ - замыкание $D$ по норме $\|\cdot\|_{-\theta}$. Поэтому оценка (8) влечет $\mathfrak{H}_{-\theta} \subset \stackrel{0}{M}[\theta]$.

Теорема 6. Пусть $\theta$ uелое, $\theta<n / 2 u \gamma>n / \theta$. Тогда

$$
H_{\gamma}^{-\theta}\left(\mathbb{R}^{n}\right) \subset \stackrel{0}{M}[\theta]
$$

и справедлива оченка

$$
\|\varphi\|_{M[\theta]} \leqslant C\|\varphi\|_{H_{\gamma}^{-\theta}} .
$$

Если $\theta=n / 2$, то аналогичное утверждение справедливо при $\gamma>2$.

ДокАЗАТЕЛЬСтво. Докажем включение

$$
H_{\gamma}^{-\theta}\left(\mathbb{R}^{n}\right) \subset M[\theta]
$$

и оценку (10). Из этой оценки и плотности линеала $D$ в $\mathfrak{H}_{-\theta}$, как и в предыдущей теореме, получим вложение (9).

Воспользуемся следующим результатом [14, гл. I, § 1.5]. Ecлu $\psi \in M\left[\mathfrak{H}_{\theta} \rightarrow \mathfrak{H}\right], m o$ для любого мультииндекса $\alpha,|\alpha| \leqslant \theta / 2$, имеем $D^{\alpha} \psi \in M[\theta]$, причем отображение $\psi \rightarrow D^{\alpha} \psi$ непрерывно.

Предположим, что уже доказано непрерывное вложение

$$
L_{\gamma}\left(\mathbb{R}^{n}\right) \subset M\left[\mathfrak{H}_{\theta} \rightarrow \mathfrak{H}\right] .
$$

Известно, что $\varphi \in H_{\gamma}^{-\theta}\left(\mathbb{R}^{n}\right)$, если и только если $\varphi=\sum_{|\alpha| \leqslant \theta} D^{\alpha} \psi_{\alpha}, \psi \in L_{\gamma}\left(\mathbb{R}^{n}\right)$. Поэтому из вложения (12) и сформулированного результата будет следовать вложение (11).

Пусть $\psi \in L_{\gamma}\left(\mathbb{R}^{n}\right)$. По условию теоремы $p:=\gamma / 2>1$. Положим $q^{-1}=1-p^{-1}$. Для функций $f \in D$ имеем

$$
\|\psi f\|_{L_{2}}=\left\|\psi^{2} f^{2}\right\|_{L_{1}} \leqslant\left\|\psi^{2}\right\|_{L_{p}}\left\|f^{2}\right\|_{L_{q}}=\|\psi\|_{L_{\gamma}}\|f\|_{L_{2 q}} .
$$

Из теоремы Соболева $\left[18\right.$, гл. 4.6.2] имеем вложение $H_{2}^{\theta} \subset L_{2 q}$, если вьполнено неравенство $n /(2 q) \geqslant n / 2-\theta$, которое эквивалентно условию теоремы $\gamma \geqslant n / \theta$. Поэтому $\|f\|_{L_{2 q}} \leqslant C\|f\|_{\theta}$ и из оценки (13) следует справедливость вложения (12), а также его непрерьвность. В случае $n=2 \theta, \gamma>2$ теорема вложения Соболева также применима и доказательство полностью сохраняется. 
TEOPEMA 7. Пусть $\theta<n / 2 u \gamma>n / 2 \theta$. Тогдa

$$
L_{\gamma}\left(\mathbb{R}^{n}\right) \subset \stackrel{0}{M}[\theta], \quad\|\varphi\|_{M[\theta]} \leqslant C\|\varphi\|_{L_{\gamma}} .
$$

Если $\theta=n / 2$, то аналогичное утверждение справедливо при $\gamma>n /(2 \theta)$.

ДокАЗАтЕльство. По условию имеем $\gamma>1$. Положим $\beta^{-1}=1-\gamma^{-1}$. Для $f \in D$, как и в теореме 7 , получаем оценки

$$
|(\varphi f, f)|=\left\|\varphi f^{2}\right\|_{L_{1}} \leqslant\|\varphi\|_{L_{\gamma}}\|f\|_{L_{2 \beta}} \leqslant C\|\varphi\|_{L_{\gamma}}\|f\|_{\theta},
$$

если вьполнено неравенство $n /(2 \beta) \geqslant n / 2-\theta$, которое эквивалентно условию $\gamma \geqslant$ $n /(2 \theta)$. При $\theta=n / 2$ доказательство сохраняется.

Теорема 8. Пусть функиия $\varphi$ зависит от $s$ переменных и является мультипликатором из $H_{2}^{\theta}\left(\mathbb{R}^{s}\right)$ в $H_{2}^{-\theta}\left(\mathbb{R}^{s}\right)$, а функиия $\psi(y)$ зависит от $k$ переменных $и$ принадлежит $L_{\infty}\left(\mathbb{R}^{k}\right)$. Пусть $n=k+s$, а число $\theta$ челое. Тогда $\varphi(x) \psi(y) \in M[\theta]$ (в пространстве $n$ переменных) и справедлива оченка

$$
\|\varphi \psi\|_{M[\theta]} \leqslant\|\varphi\|_{M[\theta]}\|\psi\|_{L_{\infty}} .
$$

Кроме того, если $\varphi \in \stackrel{0}{M}[\theta]$ или $\varphi \in M_{0}[\theta]$, то $\varphi \psi \in M_{0}[\theta]$ в пространстве $\mathbb{R}^{n}$.

ДоказАтельство. Для функций $f(x, y) \in D\left(\mathbb{R}^{n}\right)$ норма в $\mathfrak{H}_{2}^{\theta}\left(\mathbb{R}^{n}\right)$ определяется равенством

$$
\|f(x, y)\|_{H_{2}^{\theta}\left(\mathbb{R}^{s}\right)}^{2}=\int_{\mathbb{R}^{s}} \sum_{|\alpha| \leqslant \theta}\left|\frac{\partial^{\alpha} f(x, y)}{\partial x^{\alpha}}\right|^{2} d x .
$$

Поэтому

$$
\int_{\mathbb{R}^{k}}\|f(x, y)\|_{H_{2}^{\theta}\left(\mathbb{R}^{s}\right)}^{2} \leqslant\|f(x, y)\|_{H_{2}^{\theta}\left(\mathbb{R}^{s+k}\right)} .
$$

Далее, учитьвая, что $\varphi$ - мультипликатор, получаем

$$
\left|(\varphi \psi f, f)_{\mathbb{R}^{s}}\right| \leqslant|\psi(y)|\|\varphi\|_{M[\theta]}\|f(x, y)\|_{H_{2}^{\theta}\left(\mathbb{R}^{s}\right)}^{2} .
$$

Проинтегрировав это неравенство по $\mathbb{R}^{k}$, получим оценку (14).

Если $\varphi \in M_{0}[\theta]$, то при любом $\varepsilon>0$

$$
\left|(\varphi f, f)_{\mathbb{R}^{s}}\right| \leqslant \varepsilon\|f(x, y)\|_{H_{2}^{\theta}\left(\mathbb{R}^{s}\right)}^{2}+C(\varepsilon)\|f(x, y)\|_{L_{2}\left(\mathbb{R}^{s}\right)}^{2} .
$$

Отсюда легко получаем $\varphi \psi \in M_{0}[\theta]$.

\section{4. Основная теорема и примеры.}

Teорема 9. Пусть $T=(-\Delta)^{m}+1, \mathcal{D}(T)=H_{2}^{2 m}\left(\mathbb{R}^{n}\right)=: \mathfrak{H}_{2 m}, \quad S_{0}=T+u(x)$, где $u(x)$ - полуограниченная вещественная функиия, принадлежсащая $L_{\mathrm{loc}}^{\infty}\left(\mathbb{R}^{n}\right)$, $\mathcal{D}\left(S_{0}\right)=D\left(\mathbb{R}^{n}\right)$, a $S$ - расиирение по Фридрихсу оператора $S_{0}$. Пусть $q(x) \in M_{0}[\mathrm{~m}]$ или $M[m]$, но нижняя существенная грань $q$ больше -1 . Тогда $S \widetilde{+} q(x)$ корректно определен как самосопряженный оператор в $\mathfrak{H}$, причем области определения квадратичных форм операторов $S$ и $S \widetilde{+} q(x)$ совпадают. Для любой последовательности гладких функиий $q_{n}(x)$, сходящихся $\kappa q(x)$ в пространстве $M[m]$, операторы $S+q_{n}$ сходятся $x S \widetilde{+} q$ в смысле равномерной резольвентной сходимости, причем спектры аппроксимирующих операторов сходятся $к$ спектру предельного. Если $q(x) \in \stackrel{0}{M}[m]$, то непрерывные спектры $S$ и $S \widetilde{+} q$ совпадают. 
ДокАЗАТЕЛЬСТво. Сформулированные утверждения следуют из теорем 2-4. Напомним лишь, что пространство $\stackrel{0}{M}[\mathrm{~m}]$ состоит из вполне непрерывных мультипликаторов.

Полученные в п. 3 результаты позволяют привести много нетривиальных примеров, в которых условия теоремы 10 выполняются.

ПРИмеР 1. Пусть $T=-d^{2} / d x^{2}$ в пространстве $\mathfrak{H}=L_{2}\left(\mathbb{R}^{1}\right)$, а $Q$ - оператор умножения на функцию $q(x) \in H_{2}^{-1}\left(\mathbb{R}^{1}\right)$. В силу теоремы $6 q \in \stackrel{0}{M}[1]$. Поэтому корректно определено расширение по Фридрихсу $T \widetilde{+} q$ оператора $-d^{2} / d x^{2}+q(x)$, заданного на $C_{0}^{\infty}\left(\mathbb{R}^{1}\right)$ квадратичной формой. Непрерьвньй спектр $T \widetilde{+} q$ совпадает $\mathrm{c} \mathbb{R}^{+}$. Для любой последовательности функций $q_{n} \in C_{0}^{\infty}(\mathbb{R})$, сходящихся к $q$ по норме $H_{2}^{-1}$, "классические" операторы Штурма-Лиувилля $T+q_{n}$ сходятся к $T \widetilde{+} q$ в смысле равномерной резольвентной сходимости.

Отметим, что более детальньй анализ (основанный на других методах) оператора Штурма-Лиувилля с сингулярными потенциалами на оси и отрезке предложен в работе [19], которая вьполнялась одновременно с этой работой.

ПрИмЕР 2. Пусть $\mathfrak{H}=L_{2}\left(\mathbb{R}^{n}\right), T(-\Delta)^{m}+1, Q=\delta(x)$, где $(\delta(x), f)=f(0)$. Из теоремы вложения Соболева имеем $H_{2}^{m}\left(\mathbb{R}^{n}\right) \subset C\left(\mathbb{R}^{n}\right)$, если $m>n / 2$, причем вложение компактно. Поэтому при любых $\varepsilon>0, f \in D$ имеем

$$
(\delta(x) f, f)=\left(\delta(x),|f|^{2}\right)=|f(0)|^{2} \leqslant \varepsilon\|f\|_{m}^{2}+C(\varepsilon)\|f\|^{2} .
$$

Так как носитель $\delta(x)$ компактен, то $\delta(x)$ - вполне непрерьвньй мультипликатор в $M[m]$, если только $m>n / 2$. Формулировку следствий из этого факта оставляем читателям.

ПримеР 3. Пусть $T=-\Delta+1$ в $L_{2}\left(\mathbb{R}^{n}\right)$, а обобщенная функция $\delta_{\Gamma, q}$ задана равенством

$$
\delta_{\Gamma, q}(f)=\int_{\Gamma} q(y) f(y) d y, \quad f(x) \in D .
$$

Здесь $y \in \mathbb{R}^{n-1}, x=(y, t) \in \mathbb{R}^{n}, \Gamma$ - гиперплоскость в $\mathbb{R}^{n}$ определяется равенством $t=0, \operatorname{ar} q(y) \in L_{\infty}\left(\mathbb{R}^{n-1}\right)$. Очевидно,

$$
\delta_{\Gamma, q}(x)=\delta(t) q(y)
$$

Из теоремы 5 следует, что $\delta(t) \in M_{0}[1]$. Из теоремы 8 тогда получаем $\delta_{\Gamma, q} \in M_{0}[1]$. Поэтому сумма $-\Delta \widetilde{+} \delta_{\Gamma, q}$ корректно определена и справедливы другие утверждения, вытекающие из теоремы 9.

Заметим, что вместо плоскости в качестве Г можно рассмотреть гладкую компактную поверхность коразмерности 1. С помощью разбиения единицы на поверхности $\Gamma$ и замены координат, вьпрямляющей поверхность, функцию $\delta_{\Gamma, q}$ можно представить в виде конечного числа слагаемых вида $(15)$, где $\Gamma$ - гиперплоскость, а $q(y)$ имеет ком-

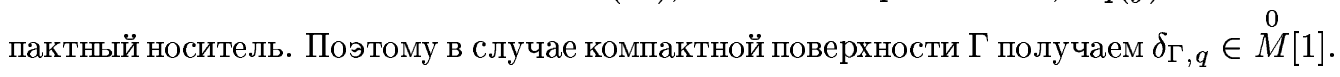


ПРимеР 4. Пусть $\Omega$ - гладкая область в $\mathbb{R}^{n}$ с компактной границей $\partial \Omega=\Gamma$. Рассмотрим функцию

$$
q_{\Gamma, \alpha}(x)=\operatorname{sign}_{\Omega}(x)(\operatorname{dist}(x, \Gamma))^{-\alpha},
$$

где $\operatorname{sign}_{\Omega}(x)=1$, если $x \in \Omega$, и -1 , если $x \notin \Omega$.

При $\alpha<2$ обобщенная функция $q_{\Gamma, \alpha}(x)$ допускает регуляризацию (см. [20])

$$
\mathrm{V} . \mathrm{P} . q_{\Gamma, \alpha}(f)=\lim _{\varepsilon \rightarrow 0} \int_{\operatorname{dist}(x, \gamma)>\varepsilon} q_{\Gamma, \alpha}(x) f(x) d x .
$$

Покажем, что при $\alpha<3 / 2$ эта функция принадлежит пространству $\stackrel{0}{M}[1]$. С помощью гладкого разбиения единицы $\sum \psi_{k} \equiv 1$ эту функцию представим в виде конечного числа функций с компактными носителями, совпадающими с $\operatorname{supp} \psi_{k}, k \geqslant 1$, и одной функции $q_{0}$ с носителем в окрестности $\infty$, причем величину $\left\|q_{0}\right\|_{L_{\infty}}$ можно сделать произвольно малой. Достаточно рассмотреть только функции $\psi_{k} q_{\Gamma, \alpha}$, для которых $\operatorname{supp} \psi_{k} \cap \Gamma \neq \varnothing$. Вблизи границы можно провести гладкую замену координат $x=(y, t)$ так, что $t=$ $\operatorname{sign}_{\Omega}(x) \operatorname{dist}(x, \Gamma)$. Тогда получим

$$
\psi_{k} q_{\Gamma, \alpha}=(\operatorname{sign} t)|t|^{-\alpha} g(y) \chi(t, y),
$$

где $\chi(t, y)$ - гладкая функция, умножение на которую не меняет мультипликативных свойств, а $g(y)$ - гладкая функция с компактным носителем.

Из теоремы 5 (при $n=1$ ) следует $|t|^{-\alpha} \operatorname{sign}(t) \in \stackrel{0}{M}[1]$, если $\alpha<3 / 2$. Из теоремы 8 и компактности носителя $g(y)$ заключаем, что $g(y)|t|^{-\alpha} \operatorname{sign}(t) \in \stackrel{0}{M}[1]$. Тем самым, при $\alpha<3 / 2$ функция (16) лежит в $\stackrel{0}{M}[1]$, операторная сумма $-\Delta \widetilde{+} q_{\Gamma, \alpha}$ определена и справедливы другие утверждения, сформулированные в теореме 9.

Основные результаты этой работы сообщались на семинарах в МГУ в 1998 году. Авторы благодарят проф. В.А.Кондратьева и А. Г. Костюченко за обсуждение результатов.

\section{СПИСОК ЦИТИРОВАННОЙ ЛИТЕРАТУРЫ}

[1] Ландау Л.Д., Лифшиц Е. М. Теоретическая физика. Т. 3. Квантовая механика. Нерелятивистская теория. М.: Наука, 1989.

[2] Березин Ф. А., Фаддеев Л. Д. Замечания об уравнении Шрёдингера с сингулярным потенциалом // Докл. АН СССР. 1961. Т. 137. № 7. С. 1011-1014.

[3] Минлос Р. А., Фаддеев Л. Д. О точечном взаимодействии для системы из трех частиц в квантовой механике // Докл. АН СССР. 1961. Т. 141. №6. С. 1335-1338.

[4] Березин Ф. А. О модели Ли // Матем. сб. 1963. Т. 60. № 4. С. 425-446.

[5] Gesztezy F., Simon B. Rank one perturbations at infinite coupling // J. Funct. Anal. 1995. V. 128. P. 245-252.

[6] Kiselev A., Simon B. Rank one perturbations with infinitesimal coupling // J. Funct. Anal. 1995. V. 130. P. 345-356.

[7] Фрагела А. К. О возмущении полигармонического оператора потенциалами с малыми носителями // Докл. АН СССР. 1979. Т. 245. №1. С. 34-36.

[8] Шондин Ю. Г. Возмущения на тонких множествах высокой коразмерности эллиптических операторов и теория расширений в пространстве с индефинитной метрикой // Зап. научн. сем. Ст. -Петерб. отдел. мат. инст. им. Стеклова (ПОМИ). 1995. Т. 222. Исследования по линейным операторам и теории функций. № 23. С. 246-292, 310-311. 
[9] Koshmanenko V., Karwowski W., Ota S. Schrödinger operator perturbed by operators related to null-sets // Positivity. 1998. V. 2. № 1. P. 77-99.

[10] Albaverio S., Gestezy F., Hoegh-Krohn R., Holden H. Some Exactly Solvable Models in Quantum Mechanics. New York: Springer-Verlag, 1988.

[11] Кошманенко В. Д. Сингулярные билинейные формы в теории возмущений самосопряженных операторов. Киев: Наукова Думка, 1993.

[12] Gunson J. Perturbation theory for a Sturm-Liouville problem with an interior singularity // Proc. R. Soc. London. A. 1987. V. 414.

[13] Kurasov P. On the Coulomb potentials in one dimension // J. Phys. A. 1996. V. 29. №8. P. 1767-1771.

[14] Мазья В. Г., Шапошникова Т. А. Мультипликаторы в пространствах дифференцируемых фунцкий. Л.: Изд. ЛГУ, 1986.

[15] Рид М., Саймон Б. Методы современной математической физики. Т. 2. М.: Мир, 1978.

[16] Каратаева Т. В., Кошманенко В. Д. Обобщенная сумма операторов // Матем. заметки. 1999. Т. 66. № 5. С. 671-682.

[17] Като Т. Теория возмущений линейных операторов. М.: Мир, 1972.

[18] Трибель Х. Теория функциональных пространств. М.: Мир, 1986.

[19] Савчук А. М., Шкаликов А. А. Операторы Штурма-Лиувилля с сингулярными потенциалами // Матем. заметки. 1999. Т. 66. №6.

[20] Гельфанд И. М., Шилов Г. Е. Обобщенные функции и действия над ними. Т. 1. М.: Физматгиз, 1959.

Московский государственный университет им. М.В. Ломоносова

Поступило

03.03.1999 